\title{
Immune Responses and Viral Phenotype: Do Replication Rate and Cytopathogenicity Influence Virus Load?
}

\author{
DOMINIK WODARZ* and MARTIN A. NOWAK \\ Institute for Advanced Study, Olden Lane, Princeton, New Jersey, 08540, USA
}

(Received 22 December 1998; In final form 8 April 1999)

\begin{abstract}
We use mathematical models to investigate the relationship between viral characteristics and virus load under the following immune responses: (a) CTL-mediated lysis, (b) CTLmediated inhibition of virus entry into target cells, $(c)$ CTL-mediated inhibition of virion production and $(d)$ antibody responses. We find that the rate of virus entry into target cells may generally only have a weak influence on virus load. The rate of virion production by infected cells only has a weak effect on the equilibrium number of infected cells while strongly influencing the number of free virus particles. On the other hand, viral cytopathogenicity may be a major determinant of virus load under certain types of immune responses. If there is no immune response, or if immune mediators inhibit infection of target cells, non-cytopathic viruses may attain significantly higher abundances than cytopathic ones. On the other hand, immune mediators acting on infected cells control both types of viruses with similar efficiencies. These results are used to interpret data on perforin-knockout experiments in LCMV infection and provide the basis for understanding the suppression and rise of non-syncytium (NSI) and syncytium inducing (SI) HIV phenotypes during the disease process.
\end{abstract}

Keywords: Cytopathogenicity, replication rate, virus load, immune responses, perforin-knockou mice, mathematical models

\section{INTRODUCTION}

Most mathematical models describing the in vivo dynamics of viral infections have focussed on the interaction between the virus population and lytic CTL responses (McLean and Kirkwood, 1990; McLean, 1992; Essunger and Perelson, 1994; Nowak and Bangham, 1996; deBoer and Perelson, 1998).
However, in most infections, alternative virus specific immune responses are thought to contribute to controlling the disease as well. With human immunodeficiency virus (HIV), CTLs may perform other anti-viral functions besides lysing infected cells. They may release chemokines inhibiting viral entry into susceptible target cells. Examples are macrophage inflammatory protein $1 \alpha$ and $1 \beta$ (MIP $1 \alpha$ and $1 \beta$ )

\footnotetext{
${ }^{*}$ Corresponding Author: Tel: 609-734-8048; Fax: 609-951-4438; E-mail: wodarz@ias.edu
} 
or RANTES (Cocchi et al., 1995; Zanussi et al., 1996; Gallo and Lusso, 1997). Alternatively, CTLs may secrete cytokines inhibiting virion production by infected cells, such as CTL-secreted anti-viral factor or CAF (Levy et al., 1996). CTL-secreted soluble factors are also thought to be a main defence mechanism necessary for controlling hepatitis $B$ virus (HBV) without damaging the liver (Guidotti et al., 1994a, 1994b; Guidotti et al., 1996a, 1996b; Guidotti and Chisari, 1996). In addition to CTL responses, antibodies are also important in contributing to the control of viral infections, e.g. with vesicular stomatitis virus (VSV) or influenza virus (Kagi and Hengartner, 1996).

In this paper, we incorporate these different types of immune responses into the basic model of virus infection (Anderson and May, 1979; Anderson and May, 1991; Nowak and Bangham, 1996; deBoer and Perelson, 1998) and investigate the effect of viral parameters on virus load. The two significant viral parameters in these models are the rate of virusmediated cell killing (cytopathogenicity) and the overall replication rate of the virus. Viral replication is a process involving multiple steps and we will concentrate on the rate of target cell infection as well as the rate of virion production by infected cells.

Viruses have different levels of cytopathogenicity. At one extreme, there are non-cytopathic viruses which hardly damage their target cells, such as lymphocytic choriomeningitis virus (LCMV, LehmannGrube, 1971; Moskophidis et al., 1993) or HBV (Guidotti and Chisari, 1996). On the other hand, viruses such as vaccinia virus, influenza virus, semliki forest virus or vesicular stomatitis virus are thought to be cytopathic (Dimmock and Primrose, 1994). The mechanisms underlying viral cytopathic effects depend on the exact relationship between the virus and its target cells (Dimmock and Primrose, 1994). They include degradation of cellular mRNA, competition between viral excess mRNA and cellular RNA, viral block of translation of cellular mRNA, block of virion assembly, the induction of an imbalance in the intracellular $\mathrm{NA}^{+} / \mathrm{K}^{+}$ ratio, or syncytium induction. Especially interesting is the case when a virus may evolve from being less cytopathic to being more cytopathic during the course of infection, as may be the case for HIV (Rudensey et al., 1995; Fouchier et al., 1996).

Different strains of a given virus may also have different replication rates. Examples are the LCMV strains Armstrong and Docile (Moskophidis et al., 1993, 1995; Wodarz et al., 1998), and HIV which evolves from relatively slow rates of reproduction in macrophages to fast rates of replication in $\mathrm{T}$ cells (O'Brien, 1994; Connor and Ho, 1994).

In the following sections we show that, according to mathematical models, the replication rate of the virus may only have a relatively weak effect on the equilibrium number of infected cells. The equilibrium number of free virus particles is significantly influenced by the rate of virion production but not by the rate of target cell entry. On the other hand, cytopathogenicity may significantly influence virus load under certain types of immune responses. More specifically, the degree of virus-induced cell killing is an important determinant of virus load under the pressure of an immune response inhibiting viral entry into target cells, while this may not be the case in the presence of immune responses acting on infected cells.

\section{THE BASIC MODEL OF VIRUS INFECTION}

The basis for all models that follow are the general virus infection equations (Nowak and Bangham, 1996; deBoer and Perelson, 1998). Uninfected target cells $(x)$ are produced at a rate $\lambda$ and suffer a natural death rate $d$. Infected cells $(y)$ die at a rate $(a+d)$ which is the sum of virus induced $(a)$ plus natural (d) death rates. They produce free virus particles at a rate $k$. The free virus $(\nu)$ decays at a rate $u$ and infects uninfected target cells at a rate $\beta$. The equations are given by:

$$
\begin{aligned}
& \dot{x}=\lambda-d x-\beta x \nu \\
& \dot{y}=\beta x \nu-(a+d) y \\
& \dot{\nu}=k y-u \nu
\end{aligned}
$$


If there is no virus infection, or if the basic reproductive ratio of the virus $\left(R_{0}=\lambda \beta k / d(a+d) u\right)$ is less than unity, the system settles to the trivial equilibrium described by (E1):

$$
x^{(1)}=\lambda / d, \quad y^{(1)}=0, \quad v^{(1)}=0 .
$$

Alternatively, the virus population is regulated by target cell availability, in which case the equilibrium expressions are given by (E2):

$$
\begin{aligned}
& x^{(2)}=(a+d) / \beta^{\prime}, \quad y^{(2)}=\lambda /(a+d)-d / \beta^{\prime}, \\
& v^{(2)}=k y^{(2)} / u
\end{aligned}
$$

where the viral replication parameters are summarised in $\beta^{\prime}=\beta k / u$.

Thus, if the virus is controlled by target cell availability only, a major viral parameter influencing the equilibrium virus load is its cytopathogenicity (a). Decreasing the rate of virus-induced cell death leads to a strong increase in virus load (Figure 1a).

As can be seen from the appropriate equilibrium expressions and from Figure $2 a$, the rate of target cell entry, $\beta$, does not exert a significant effect on virus load given that $R_{0}$ remains well above one (Bonhoeffer and Nowak, 1997; Bonhoeffer et al., 1997a, 1997b). For $\beta^{\prime} \gg d$ we have $y^{(2)} \approx \lambda /$ $(a+d)$. The rate of virion production by infected cells also has only a weak effect on the equilibrium number of infected cells while strongly influencing the number of free virus particles at equilibrium (Figure 3a).

\section{MODELLING CTL MEDIATED LYSIS}

Let us now consider a CTL response, $z$, which eliminates infected cells. We have

$$
\begin{aligned}
& \dot{x}=\lambda-d x-\beta x \nu \\
& \dot{y}=\beta x \nu-(a+d) y-p y z \\
& \dot{\nu}=k y-u \nu \\
& \dot{z}=\frac{c y z}{1+\varepsilon z}-b z
\end{aligned}
$$

We assume that infected cells are killed at the rate pyz. CTL are stimulated at a rate $c y z /(1+\varepsilon z)$. This is a saturating function of $z$ : at high CTL densities the rate of CTL proliferation is converging to $c y / \varepsilon$. Finally, CTL die at a rate $b z$.

If $R_{0}>1$ there are two stable equilibria; the virus may establish an infection without the presence of an immune response, being limited by target cell availability only. This outcome is described by (E4):

$$
\begin{aligned}
& x^{(4)}=(a+d) / \beta^{\prime}, \quad y^{(4)}=\lambda(a+d)-d / \beta^{\prime}, \\
& v^{(4)}=k y^{(4)} / u, \quad z^{(4)}=0
\end{aligned}
$$

This equilibrium is stable if the number of infected target cells in the absence of an immune response is below the threshold needed for an immune response to become established, i.e. if $c\left(\lambda /(a+d)-d / \beta^{\prime}\right)<b$.

Alternatively, if the above condition is violated, the virus is controlled by a combination of target cell availability and CTL mediated lysis leading to the following equilibrium (E5).

$$
\begin{aligned}
& y^{(5)}=b\left[\varepsilon \beta^{\prime} x^{(5)}-\varepsilon(a+d)+p\right] /(p c), \\
& v^{(5)}=k y^{(5)} / u, \quad z^{(5)}=\left(\beta^{\prime} x^{(5)}-a-d\right) / p,
\end{aligned}
$$

where $x^{(5)}$ is given by:

$$
x^{(5)}=\frac{\left[b \beta^{\prime}(\varepsilon a+\varepsilon d-p)-c p d\right]+}{\sqrt{\left[b \beta^{\prime}(\varepsilon a+\varepsilon d-p)-c p d\right]^{2}+4 \varepsilon \beta^{2} b \lambda p c}} .
$$

Here, the cytopathogenicity of the virus, $a$, contributes only additively to the equilibrium expression for virus load. Therefore, as shown in Figure $1 \mathrm{~b}$, cytopathogenicity is not an important determinant of virus load (except when a $\gg p$, i.e. when virusmediated cell killing is faster than CTL-mediated killing). That is, in the presence of a strong lytic CTL response, viruses characterised by a low rate of cell killing (small $a$ ) will not attain significantly higher virus loads than those characterised by larger values of $a$.

As shown in Figure $2 b$, an increase in the rate of target cell entry drives virus load up to an asymptote. Therefore, for $R_{0} \gg 1$, virus load becomes relatively independent of this parameter. Similarly, the rate of virion production, $k$, drives the equilibrium number of infected cells towards an asymptote for $R_{0} \gg 1$. 
(a) No immune response
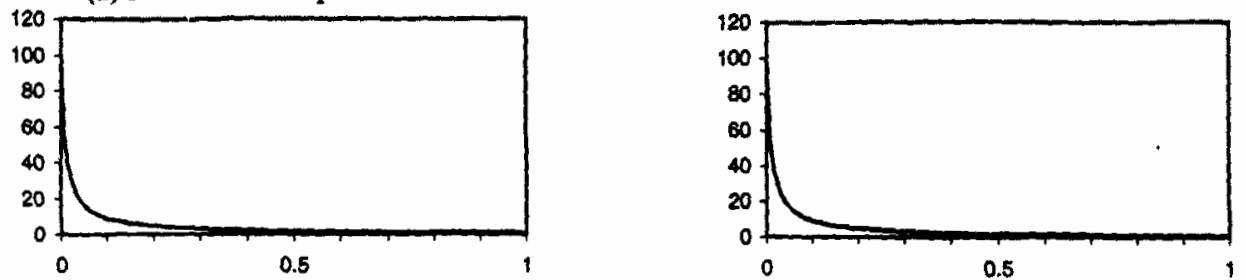

(b) CTL-mediated lysis
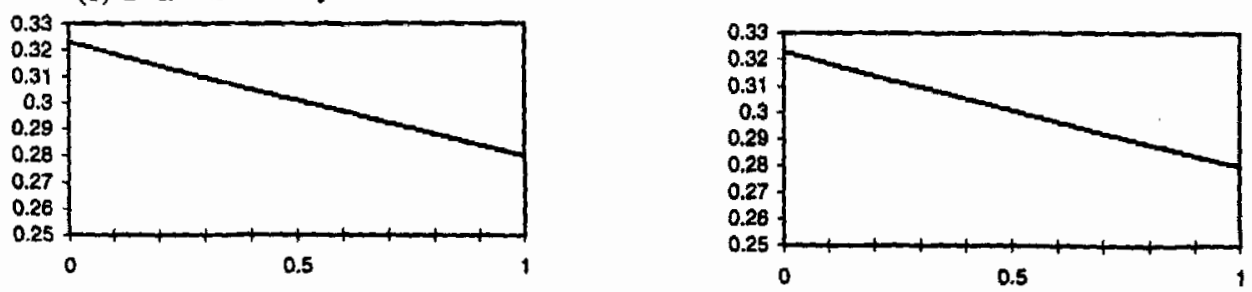

(c) CTL-mediated inhibition of virus entry
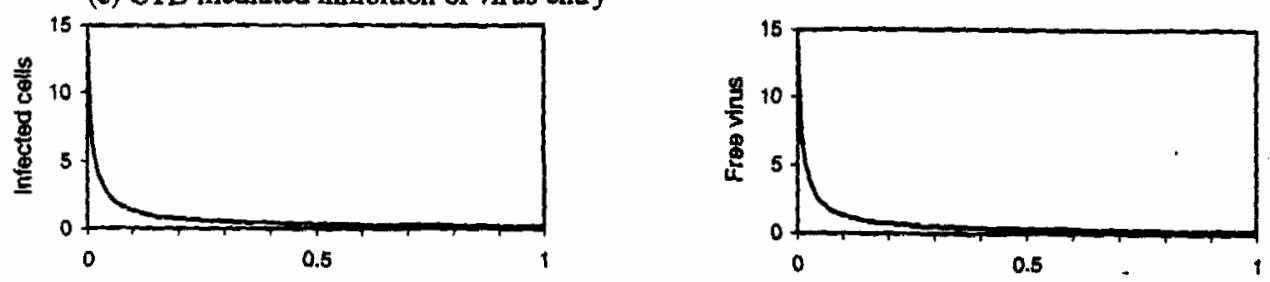

(d) CTL-mediated inhibition of virion production
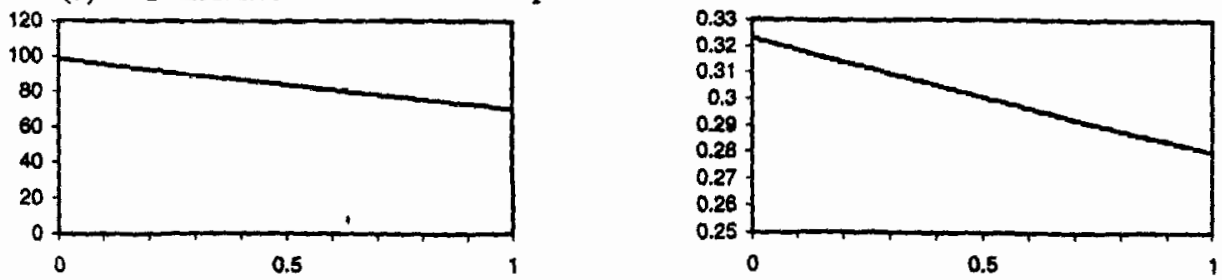

(e) Neutralising antibodies
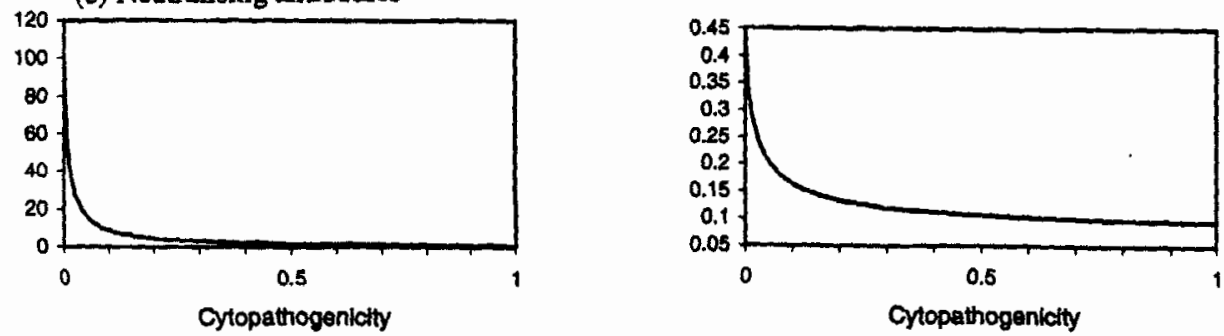

FIGURE 1 Dependence of $(i)$ the equilibrium number of infected cells and $(i i)$ free virus particles on the cytopathogenicity of the virus under different types of immune responses. (a) no immune response, (b) CTL-mediated lysis, (c) CTL mediated inhibition of virus entry, (d) CTL-mediated intibition of virion production and $(e)$ neutralising antibodies. The graphs demonstrate that no immune response or immune responses inhibiting infection of target cells (CTL-mediated inhibition of virus entry and neutralising antibody responses) favour non-cytopathic viruses, while immune mediators acting on infected cells (CTL-mediated lysis and CTL-mediated inhibition of virion production) control both types of viruses with similar efficiencies. Parameters were chosen as follows: $\lambda=1$, $d=0.01, \beta=2, k=2, u=2, c=2.5, b=0.2, \varepsilon=1$. For the lytic CTL tesponse, $p=1$, whereas for immune responses involving soluble mediators, $p=100$ since a single cell secretes many such soluble mediators and is thus more efficient at inhibiting the virus. 
(i)

(a) No immune response

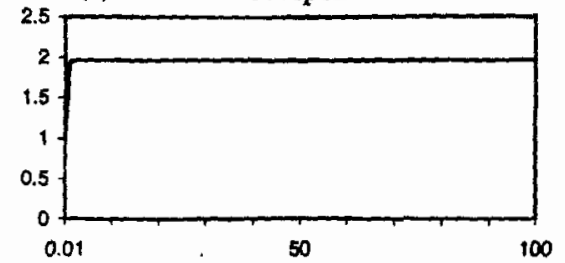

(b) CTL-mediated lysis

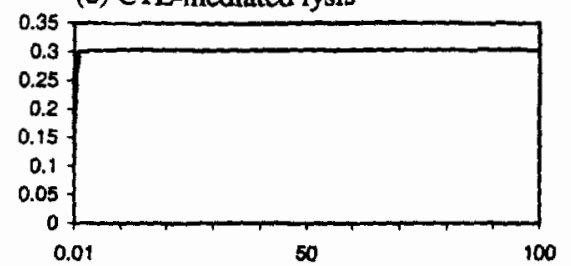

(c) CTL-mediated inhibition of virus entry

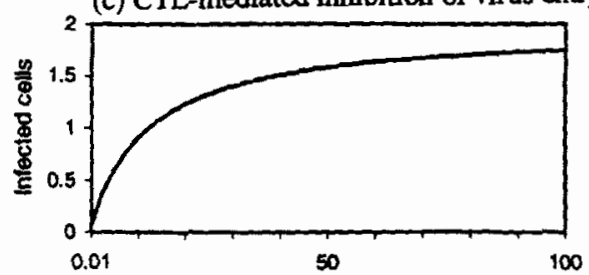

(d) CTL-mediated inhibition of virion production

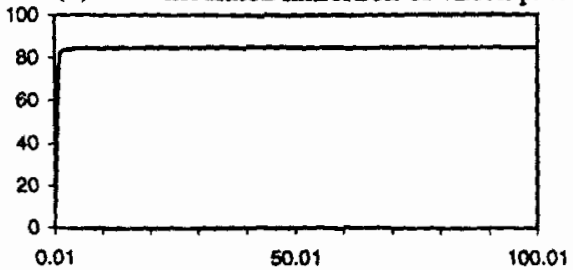

(e) Neutralising antibodies

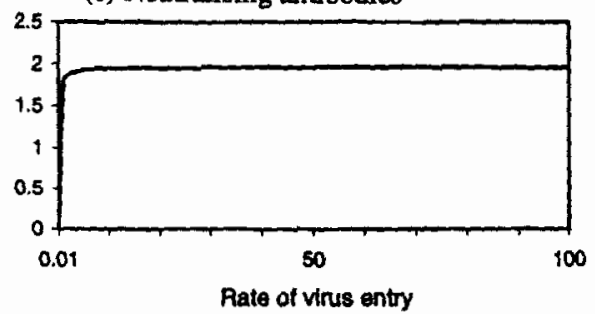

(ii)
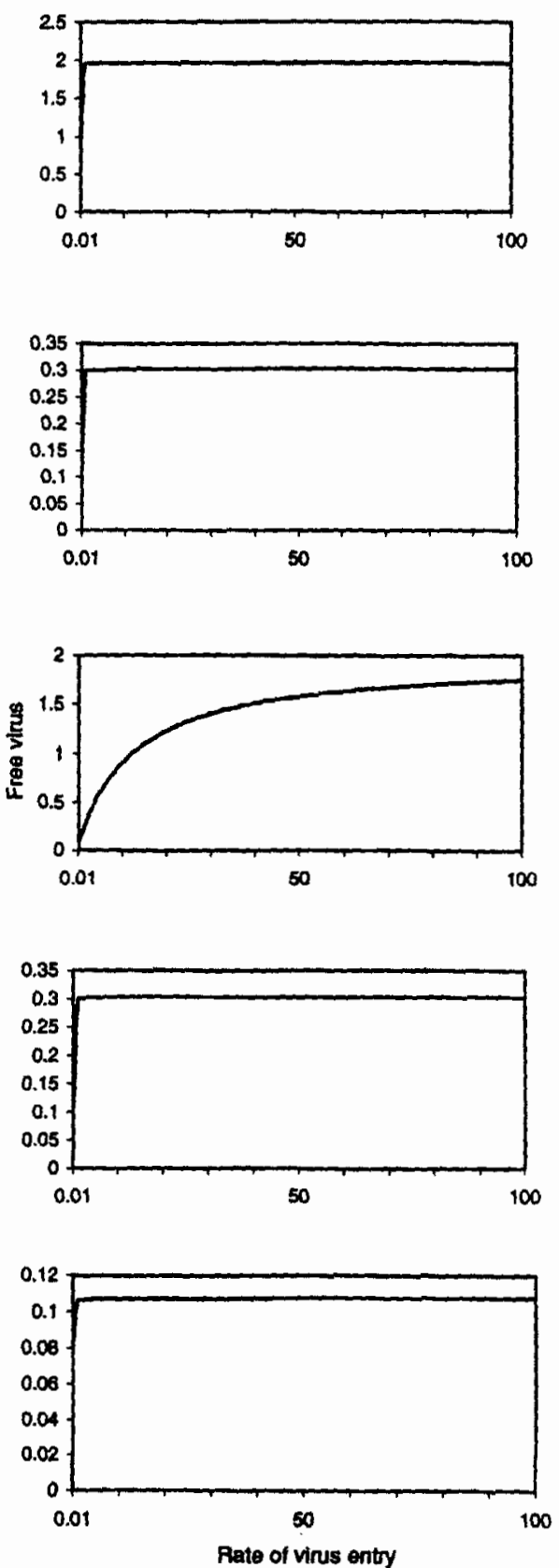

FIGURE 2 The effect of the rate of viral entry into target cells $(\beta)$ on (i) the equilibrium number of infected cells and (ii) free virus particles under different types of immune responses. (a) no immune response, (b) CTL-mediated lysis. (c) CTL mediated inhibition of virus entry, (d) CTL-mediated inhibition of virion production and (e) neutralising antibody response. The graphs show that the rate of virus entry only has a weak or no significant influence on virus load. Increasing the parameter $\beta$ drives virus load towards an asymptote. The asymptotic behaviour is reached at significantly higher values of $\beta$ if the immune response inhibits virus entry $(c)$. This is because an increase in the rate of target cell entry directly counters the immune response. Parameters were chosen as follows: $\lambda=1$, $d=0.01, a=0.5, k=2, u=2, c=2.5, b=0.2, \varepsilon=1$. For the lytic CTL response, $p=1$, whereas for immune responses involving soluble mediators, $p=100$ since a single cell secretes many such soluble mediators and is thus more efficient at inhibiting the virus. 
(i)

(a) No immune response

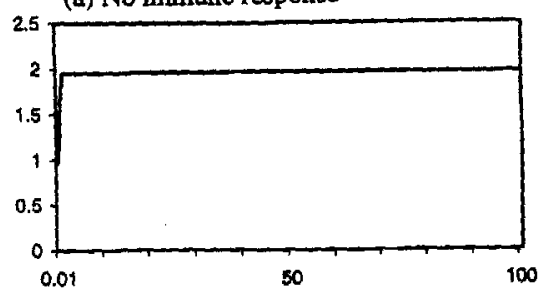

(b) CTL-mediated lysis

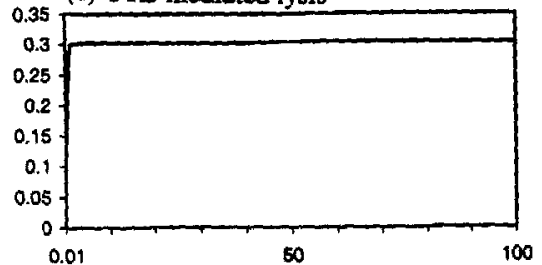

(c) CTL-mediated inhibition of virus entry

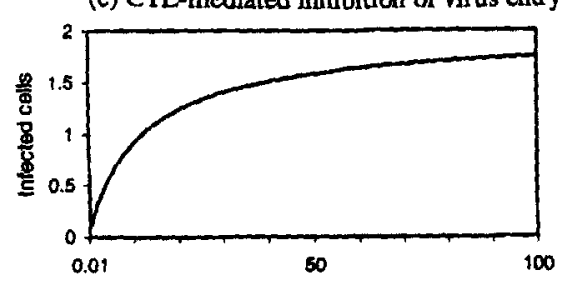

(d) CTL-mediated inhibition of virion production

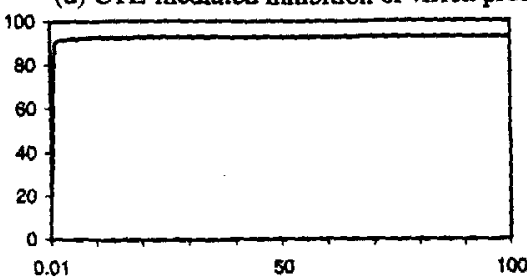

(e) Neutralising antibodies

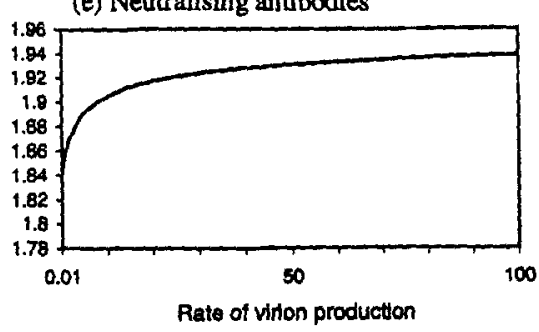

(ii)
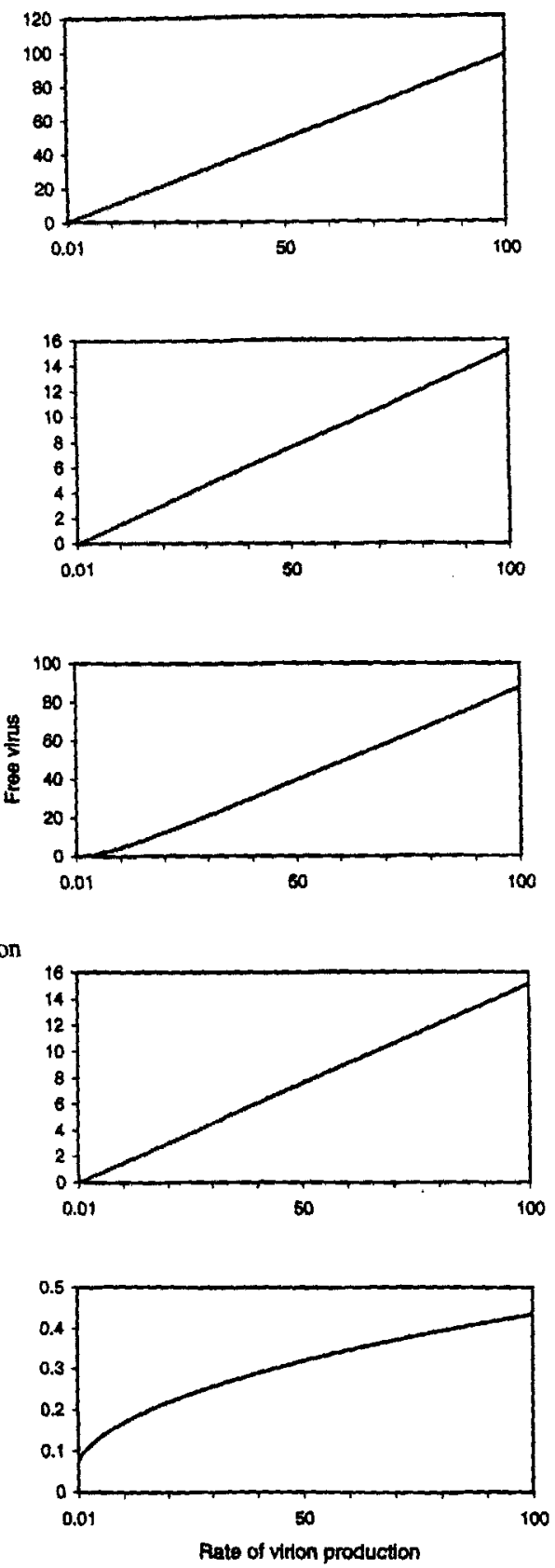

FIGURE 3 The effect of the rate of virion production $(k)$ on (i) the equilibrium number of infected cells and (ii) free virus particles under different types of immune responses. (a) no immune response, (b) CTL-mediated lysis, (c) CTL mediated inhibition of virus entry, (d) CTL-mediated inhibition of virion production and (e) neutralising antibody response. Increasing the rate of virion production drives the equilibrium number of infected cells towards an asymptote and thus only has a relatively weak effect. The parameter range over which the parameter $k$ has a stronger influence on the number of infected cells is broadened if the immune response inhibits virus entry. On the other hand, the equilibrium number of free virus particles is significantly influenced by the rate of virion production. Parameters were chosen as follows: $\lambda=1, d=0.01, \beta=2, a=0.5, u=2, c=2.5, b=0.2, \varepsilon=1$. For the lytic CTL response, $p=1$, whereas for immune responses involving soluble mediators, $p=100$ since a single cell secretes many such soluble mediators and is thus more efficient at inhibiting the virus. 
As before, this parameter significantly influences the number of free virus particles (Figure $3 b$ ).

\section{MODELLING CYTOKINE MEDIATED VIRUS INHIBITION BY CTLS}

There are two basic modes in which cytokines may limit virus replication. They may inhibit infection of target cells, or they may inhibit the process of virion production itself by destroying viral DNA or RNA. Models for the respective scenarios will be considered in turn.

\section{(a) Block of Viral Infection:}

Cytokine mediated inhibition of virus entry into target cells may be modelled by the following set of differential equations:

$$
\begin{aligned}
& \dot{x}=\lambda-d x-\frac{\beta x \nu}{p z+1} \\
& \dot{y}=\frac{\beta x \nu}{p z+1}-(a+d) y \\
& \dot{\nu}=k y-u \nu \\
& \dot{z}=\frac{c y z}{1+\varepsilon z}-b z
\end{aligned}
$$

$\mathrm{H}$ re, $z$ denotes the cytokine producing CTL population. The rate of target cell infection is given by $\beta x \nu /(p z+1)$; thus an increased abundance of CTL, $z$, reduces target cell entry. Cells that do become infected produce new virus particles at an uncompromised rate $k$.

This system is also characterised by equilibrium (E4) if $c\left(\lambda /(a+d)-d / \beta^{\prime}\right)<b$. Alternatively, the virus is controlled by a combination of target cell availability and cytokine action, which is described by (E6).

$$
\begin{aligned}
& x^{(6)}=\frac{\lambda p c+b(a+d)(\varepsilon-p)}{d p c+\varepsilon b \beta^{\prime}}, \\
& y^{(6)}=\frac{b\left[\varepsilon \beta^{\prime} \lambda-d(a+d)(\varepsilon-p)\right]}{(a+d)\left(d p c+\varepsilon b \beta^{\prime}\right)}, \\
& \nu^{(6)}=k y^{(6)} / u, \quad z^{(5)}=\frac{\lambda c \beta^{\prime}-(a+d)\left(b \beta^{\prime}+d c\right)}{(a+d)\left(d p c+\varepsilon b \beta^{\prime}\right)}
\end{aligned}
$$

In contrast to the model for CTL mediated lysis of infected cells, the rate of virus induced cell death (a) is an important factor influencing the equilibrium virus load (Figure 1c). Since the death rates of target cells form a factor in the denominator of the equilibrium expression for virus load, decreasing the rate of cell death leads to a strong increase in virus load. Thus, whereas cytopathic viruses (relatively large a) are kept at low equilibrium levels, non-cytopathic viruses (small $a$ ) attain much higher abundances and are thus less efficiently controlled by CTL-mediated inhibition of virus infection.

Again, an increase in the rate of target cell entry, $\beta$, pushes the equilibrium virus load against an asymptote, thus only exerting a weak influence above a certain value of $R_{0}$ (Figure $2 \mathrm{c}$ ). However, as can be seen in Figure $2 c$, the asymptotic behaviour is only reached at relatively high rates of target cell entry, resulting in a stronger influence of $\beta$ on virus load over a wider parameter range. The reason for this is that the immune response inhibits viral entry. Consequently, increasing the parameter $\beta$ directly counters this immune response. The same applies to the effect of the rate of virion production on the equilibrium number of infected cells. Again, the rate of virion production significantly influences the number of free virus particles.

\section{(b) Inhibition of Virion Production}

Here we model the situation in which CTL-secreted cytokines limit the rate of virion production inside the host cell. In this case, the rate of CTL proliferation cannot simply be proportional to the number of infected cells, since a reduction in virion production will lead to a reduction of the amount of antigen presented on the surface of an infected cell. Therefore, we let the rate of CTL proliferation be proportional to $y /(p z+1)$ instead of just $y$. Moreover, the rate of virus induced cell death also has to become a function of the amount of CTLs present. The higher the levels of CTLs, the fewer particles causing cytopathogenicity will be produced by an infected cell which will therefore have a longer life span. These assumptions lead to the following set of 
equations:

$$
\begin{aligned}
& \dot{x}=\lambda-d x-\beta x \nu \\
& \dot{y}=\beta x \nu-\left(d+\frac{a}{p z+1}\right) y \\
& \dot{\nu}=\frac{k y}{p z+1}-u \nu \\
& \dot{z}=\frac{c y z}{(p z+1)(1+\varepsilon z)}-b z
\end{aligned}
$$

This model again shows virus infection without the presence of a CTL response (E4). If virus load in the absence of an immune response is above the threshold needed for the immune system to become activated, the equilibrium expressions are given by (E7).

$$
\begin{aligned}
& \lambda c p d+a b[\varepsilon(a+d)-d p] \\
& y^{(7)}= \frac{-x^{(7)}\left(\varepsilon a b \beta^{\prime}+c d^{2} p\right)}{c d^{2} p}, \\
& \nu^{(7)}= \frac{k b\left[\varepsilon\left(\beta^{\prime} x^{(7)}-d-a\right)+d p\right]}{u c d p} \\
& z^{(7)}= \frac{\left(\beta^{\prime} x^{(7)}-d-a\right)}{p d}
\end{aligned}
$$

where $x^{(7)}$ is given by the solution of a quadratic equation:

$$
x^{(7)}=u \frac{\sqrt{\begin{array}{c}
{[\varepsilon \beta k b(d+a)-p d(\beta k b+d u c)]^{2}} \\
+4 \varepsilon b \beta^{2} k^{2} \lambda c d p
\end{array}}}{2 \varepsilon b \beta^{2} k^{2}}
$$

The dependence of the equilibrium virus load on the cytopathogenicity of the virus is different compared to the previous mode of cytokine action. The degree of target cell killing again contributes only additively to the equilibrium expressions. Therefore, viral cytopathogenicity, $a$, is not an important determinant of virus load (unless virus-mediated cell killing occurs at a faster rate than CTL-mediated inhibition, $a \gg p$ ). That is, in the presence of effective CTL-mediated inhibition of virion production, non-cytopathic viruses (small $a$ ) will not attain significantly higher loads than viruses characterised by larger rates of cell killing (larger $a$, Figure 1d).
The rate of target cell entry, $\beta$, does not have a significant influence on virus load above a certain value of $R_{0}$. (Figure $2 \mathrm{~d}$ ). The rate of virion production, $k$, also shows only a weak effect on the equilibrium number of infected cells (Figure 3d). Although in this case the immune response again directly counters viral replication, the parameter range over which $\beta$ and $k$ significantly influence virus load and the number of infected cells, respectively, is not broadened as was the case in the last section. The reason is that in this case the immune system inhibits the rate of virion production which does not directly contribute to the infection of new target cells. As before, the equilibrium number of free virus particles is significantly influenced by the rate of virion production (Figure $3 \mathrm{~d}$ ).

\section{MODELLING ANTIBODY RESPONSES}

The antibody response is modelled in a similar way as the CTL response. The main difference is that antibody secreting $B$ cells are activated by antigen specific $\mathrm{CD}^{+} \mathrm{T}$ cells which recognize viral antigen on the surface of antigen presenting cells (APCs) such as macrophages or interdigitating dendritic cells. Since the amount of antigen presentation by the APCs is proportional to the abundance of free virus particles, the growth of this immune response must be proportional to $v$ rather than to $y$. Once the antibody response has developed it removes free virus particles a rate $p$. Denoting the antibody response by $z$, the model is written as

$$
\begin{aligned}
& \dot{x}=\lambda-d x-\beta x v \\
& \dot{y}=\beta x v-(a+d) y \\
& \dot{v}=k y-u v-p v z \\
& \dot{z}=\frac{c v z}{1+\varepsilon z}-b z
\end{aligned}
$$

If $R_{0}>1$ and $c[(\lambda k) /(a u)-d / \beta]<b$, virus control by target cell availability (E4) will be observed. The equilibrium expressions describing virus control by a combination of target cell availability and 
the antibody response are given by (E8)

$$
\begin{aligned}
y^{(8)} & =\left(\lambda-d x^{(8)}\right) /(a+d), \\
v^{(8)} & =\frac{\left[b\left\{\varepsilon \beta k x^{(8)}+(a+d)(p-\varepsilon u)\right\}\right]}{[(a+d) p c]}, \\
z^{(8)} & =\left[\beta k x^{(8)}-u(a+d)\right] /[p(a+d)],
\end{aligned}
$$

where $x^{(8)}$ is obtained by a solution of a quadratic equation

$$
x^{(8)}=\frac{(a+d)[\beta b(u \varepsilon-p)-d p c]+}{\sqrt{\{(a+d)[\beta b(u \varepsilon-p)-d p c]\}^{2}}+4 \varepsilon(a+d) \beta^{2} b k \lambda p c}-2
$$

As is apparent from these expressions as well as from Figure 1e, low degrees of cytopathogenicity strongly increase virus load. Thus, as was the case with cytokine mediated inhibition of virus entry into target cells, antibody responses are less effective at controlling non-cytopathic viruses than cytopathic ones.

As was the case with the CTL responses, an increase in the rate of target cell entry drives the equilibrium virus load towards an asymptote (Figure 2e), and the same is true for the effect of increasing the rate of virion production on the equilibrium number of infected cells. The rate of virion production again exerts a strong effect on the number of free virus particles.

\section{APPLICATION AND DISCUSSION}

We have analysed a series of mathematical models describing the dynamics between a virus population and one of four alternative immune responses: CTLmediated lysis, CTL-mediated inhibition of virus entry into target cells, CTL-mediated inhibition of virion production, and an antibody response. Essentially, two viral parameters influence virus load at equilibrium: the replication rate of the virus and the degree of virus-induced target cell killing. Our models have shown that the rate of target cell entry only has a weak influence on virus load at equilibrium given that $R_{0} \gg 1$. This is because an increase in the parameter $\beta$ drives virus load towards an asymptote. Thus, above a certain value of $\beta$, virus load will become relatively independent of this parameter. The parameter range over which the rate of target cell entry has a stronger effect on virus load is broadened if the immune system directly inhibits the process of viral entry. The same considerations apply to the effect of the rate of virion production on the equilibrium number of infected cells. In contrast, the models indicate that the rate of virion production always has a significant influence on the number of free virus particles. If patients differ in their rate of virion production, $k$, the number of infected cells may not correlate with the number of free virus particles in cross-sectional studies.

The degree of virus-mediated cell killing may have a significant influence on virus load at equilibrium under certain types of immune responses. In the absence of an immune response, non-cytopathic viruses will clearly achieve significantly higher abundances of infected cells than more cytopathic ones. Similarly, immune responses inhibiting viral entry into target cells (CTL-mediated inhibition of virus entry and antibodies) only have a weak effect on non-cytopathic viruses. On the other hand, immune responses acting on infected cells (CTL-mediated lysis and CTL-mediated inhibition of virion production) have a strong effect on non-cytopathic viruses.

The mechanisms underlying these phenomena are as follows. Without any immune response, cells infected with non-cytopathic viruses will have a longer life span than cells infected with cytopathic viruses and will thus be able to produce more virus particles leading to high virus loads, given that the rate of virion production, $k$, is the same. If the immune system inhibits viruses from entering their target cells but does not inhibit virion production once a host cell has been successfully entered, a cell will again produce many more virus particles during its life span when harbouring a non-cytopathic, compared to a cytopathic virus, given that there is no difference in the rate of virion production. Similar considerations apply to the interactions between the virus and an antibody response. The situation is different with immune responses acting on infected 
cells. A lytic CTL response will significantly reduce virus load of a non-cytopathic virus since it shortens the life span of an infected cell and thus reduces the amount of virus particles produced by that cell. On the other hand, for a cytopathic virus, a lytic CTL response may not have a significant impact, since the virus itself already destroys the cell. With CTLmediated inhibition of virion production the mechanism is different. If cytokines limit the amount of virion production, they also limit the amount of virus induced target cell damage, since viral cytopathic effects are usually a by-product of viral replication. Thus, the life-span of infected cells harbouring cytopathic and non-cytopathic viruses, and therefore the amount of virus produced during that life-span, will be similar.

The result that the rate of viral entry into target cells only has a weak influence on virus load is counter-intuitive and this issue has never been addressed experimentally. However, this phenomenon has repeatedly been observed in mathematical models of viral infections (Bonhoeffer and Nowak, 1997; Bonhoeffer ef al., 1997a, 1997b). The finding that immune responses directly inhibiting the process of viral entry widen the parameter range over which virus load may more strongly depend on the rate of viral replication might have importance for understanding the dynamics of drug-treatment in HIV-infection. If the virus is not eliminated, application of reverse transcriptase inhibitors or protease inhibitors have been shown to lead to significant reductions in virus load. However, Bonhoeffer et al. (1997a) showed that the relative independence of virus load on the replication kinetics of the virus in mathematical models makes this observation difficult to explain unless the strength of a lytic CTL response is very high. Chemokines inhibiting virus entry into target cells are thought to be an important mechanism controlling HIV in the asympromatic period (Levy et al., 1996). Therefore, the presence of these immune mechanisms might contribute to the fall in virus load upon drug-treatment in addition to the factors listed by Bonhoeffer et al. (1997a).

On the other hand, experiments have addressed the effect of viral cytopathogenicity on virus load under different types of immune responses. The key experiments in this context are perforin-knockout studies. CTL-mediated cytotoxicity may be achieved by two pathways (Kagi et al., 1995a, 1995b; Kagi et al, 1996). Although the interaction of the Fas molecule on the surface of the $T$ cell with the Fas ligand on the target cell can induce apoptosis in the target cell, the main effector mechanism for CTL-mediated lysis in vivo is supposed to be the secretion of the pore-forming molecule perforin by the $\mathrm{CD}^{+}$cell. Consequently, an important tool for studying the significance of CTL-mediated lysis for controlling viral infections has been the use of perforin-knockout mice. While this defect does not restrict the activation and proliferation of $\mathrm{CD}^{+}$ cells, such mice are unable to lyse infected cells by the perforin-dependent pathway (Kagi et al., 1994). Any control of the virus in these mice must therefore be mainly due to soluble immune mediators, such as antibodies or cytokines/chemokines inhibiting virion production or entry into target cells.

\section{(a) Experimental Observations}

It has been shown that perforin-knockout mice do not lose control of cytopathic viruses such as vaccinia, vesicular stomatitis, semliki forest, or influenca virus, but control them with similar efficiencies as wild-type mice (Kagi et al., 1995a, 1995b; Kagi et al., 1996; Kagi and Hengartner, 1996). On the other hand, perforin-knockout mice infected with the non-cytopathic LCMV have been reported to be severely compromised in their ability to control the infection (Kagi et al., 1994; Walsh et al., 1994; Kagi et al., 1995a, 1995b; Kagi et al., 1996; Kagi and Hengartner, 1996; Zinkernagel, 1996). Based on these observations, Kagi and others formulated the hypothesis that CTL-mediated lysis is an essential immune mechanism for fighting non-cytopathic viruses in general, whereas soluble immune factors are sufficient to combat cytopathic viruses.

However, HBV, another non-cytopathic virus, forms an exception to this scheme. Perforin-mediated lysis is not necessary in order to control HBV infection and it has been shown in a transgenic mouse 
model that CTL-secreted soluble factors, mainly IFN$\gamma$ and TNF- $\alpha$, are responsible for keeping the virus population in check (Guidotti et al., 1994a, 1994b; Chisari and Ferrari, 1995; Tsui et al., 1995; Guidotti and Chisari, 1996; Guidotti et al., 1996a, 1996b; Cavanaugh et al., 1997; Chisari 1997). They demonstrated that these cytokines induce $\mathrm{HBV}$ infected hepatocytes to inhibit virion production at a posttranscriptional stage (Tsui et al., 1995; Guidotti et al., 1996b), mainly through destabilising viral RNA and destroying HBV nucleocapsid particles (Guidotti et al., 1996b). It is not clear whether CTL-mediated cytokine action is able to eliminate the viral infection, i.e. to cure infected cells, or whether the viral genome persists in some form in the hepatocytes (Guidotti et al., 1996b). Studies reporting the persistence of $\mathrm{HBV}$ traces as well as persistence of activation markers on HBV-specific CTLs many years after recovery from acute hepatitis (Michalak et al., 1994; Rehermann et al., 1995; Rehermann et al., 1996) argue against clearance of the HBV genome.

\section{(b) Simulations}

Based on the mathematical models analysed in this paper, we have simulated such perforin-knockout experiments assuming that the main alternative to CTL mediated lysis is given by (i) CTL-mediated inhibition of virus infection, (ii) neutralising antibodies, and (iii) CTL-mediated inhibition of virion production (Table I). We investigate which of the alternative immune responses may lose control of non-cytopathic viruses in perforin-knockout compared to perforin-competent mice. As shown in Figure 4, perforin-knockout mice significantly lose control of non-cytopathic viruses given that the alternative immune response is inhibiting viral entry, i.e. CTL-mediated inhibition of virus infection and antibody responses. On the other hand, with immune responses acting on infected cells (CTL-mediated inhibition of virion production), there is no significant loss of control of non-cytopathic viruses in perforin knockout mice.

\section{(c) Interpretation}

The above described simulations demonstrate that our mathematical models can reconcile the apparently conflicting observations on the control of LCMV and HBV infection in perforin-knockout mice. Both the observations on LCMV and HBV do not contradict the theoretical results presented here.

TABLE I Mathematical models used for simulating perforin knockout experiments assuming that the main aiternative to a ivtic CTL response is given by (a) CTL-mediated inhibition of virus entry, (b) CTL-mediated inhibition of virion production and (c) neutralising antibodies. In the latter case, we denote the antibody response by $z_{1}$ and the lytic CTL response by $z_{2}$. The rate of CTL-mediated lysis is described by the parameter $p$, while the rate of virus inhibition by the alternative immune response is given by the parameter $q$. When the alternative immune response is cytokine-mediated inhibition of virion production, the rate of target cell killing by the lytic CTL response must be proportional to $1 /(q z+1)$, since cytokine action reduces the amount of antigen displayed on the surface of the infected cells and therefore the rate of recognition and killing by the CTLS

(a)

$$
\begin{aligned}
& \dot{x}=\lambda-d x-\frac{\beta x \nu}{q z+1} \\
& \dot{y}=\frac{\beta x \nu}{q z+1}-(a+d) y-p y z \\
& \dot{\nu}=k y-u \nu \\
& \dot{z}=\frac{c y z}{\varepsilon z+1}-b z
\end{aligned}
$$

(b)

(c)

$$
\begin{aligned}
& \dot{x}=\lambda-d x-\beta x \nu \\
& \dot{y}=\beta x \nu-\left(d+\frac{a}{q z+1}\right) y-\frac{p y z}{q z+1} \\
& \dot{\nu}=\frac{k y}{q z+1}-u \nu \\
& \dot{z}=\frac{c y z}{(q z+1)(\varepsilon z+1)}-b z
\end{aligned}
$$

$$
\begin{aligned}
& \dot{x}=\lambda-d x-\beta x \nu \\
& \dot{y}=\beta x \nu-(a+d) y-p y z_{2} \\
& \dot{\nu}=k y-u \nu-q \nu z_{1} \\
& \dot{z}_{1}=\frac{c \nu z_{1}}{\varepsilon z_{1}+1}-b z_{1} \\
& \dot{z}_{2}=\frac{c y z_{2}}{\varepsilon z_{2}+1}-b z_{2}
\end{aligned}
$$


(a) CTL-mediated inhibition of virus entry

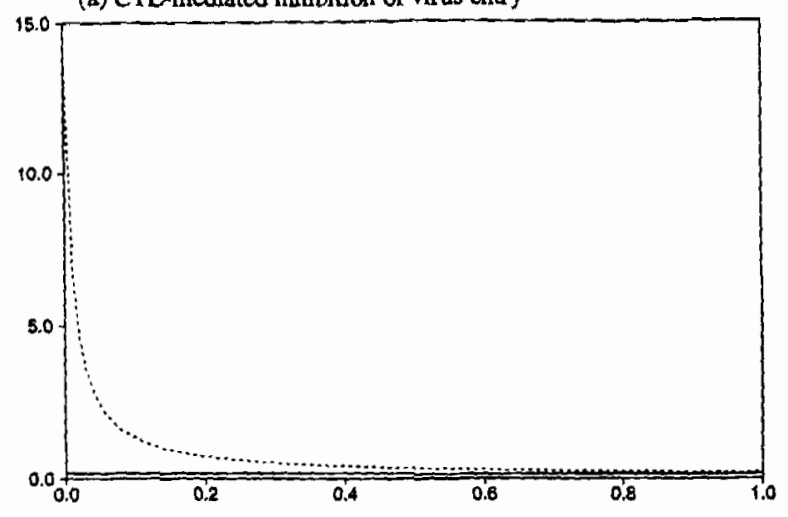

(b) Neutralising antibodies

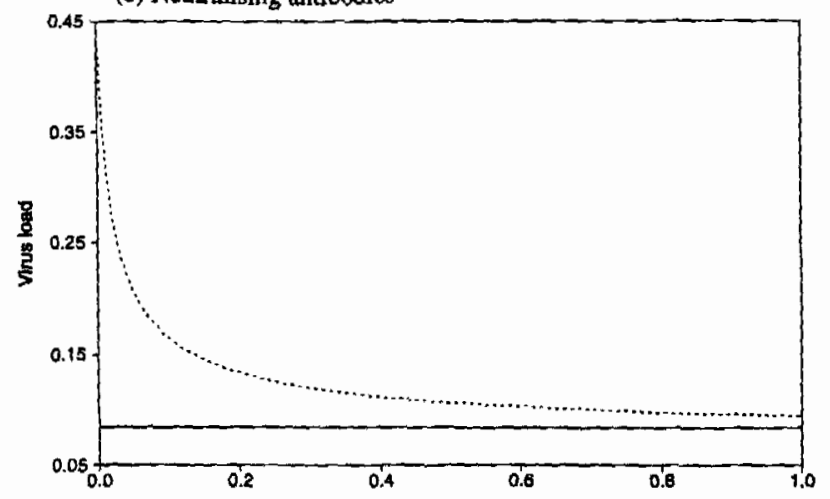

(c) CTL-mediated inhibition of virion production

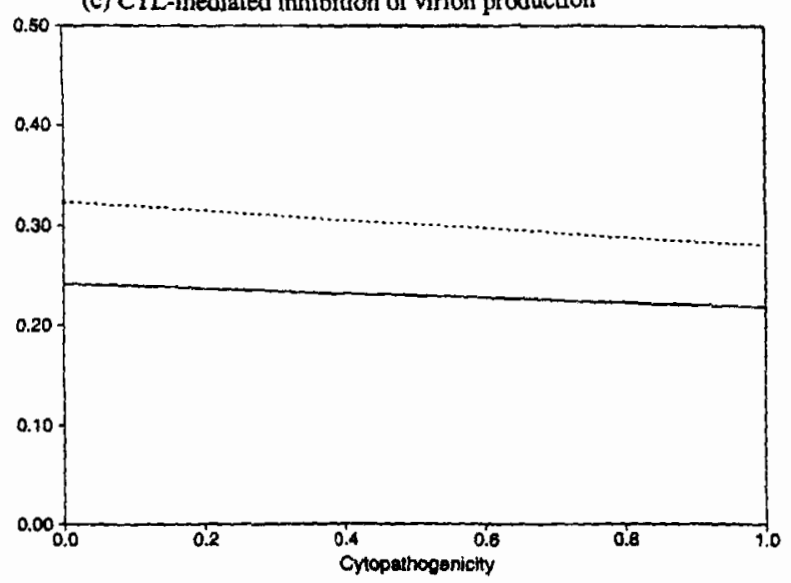

FIGURE 4 Perforin-knockout experiments simulation. Virus load in wild-type and perforin-knockout simulations is compared assuming that in the perforin-knockout simulations the main alternative immune response to CTL-mediated lysis is (a) CTL-mediated ithibition of virus entry, (b) neutralising antibodies, and (c) CTL-mediated inhibition of virion production. Perforin knockout mice significantly lose control of non-cytopathic viruses if the alternative immune response is inhibition of virus entry or an antibody response, while this is not the case if the alternative immune response is CTL mediated inhibition of virion production. The equations underlying these simulations are set out in Table $I$ and parameters were chosen as follows: $\lambda=1, d=0.01, \beta=2, k=2, u=2, c=2.5$, $b=0.2, q=100, \varepsilon=1$. For perforin-competent mice, $p=1$, for perforin-knockout mice $p=0$. 
Our models predict that the non-lytic CTL response in HBV does not lose control of the virus due to its non-cytopathic nature. This is because, as discussed above, CTL-secreted cytokines induce inhibition of virion production inside the infected cell. The relevance of the different kinds of alternative immune responses for controlling LCMV infection in perforin-knockout mice is less clear. Secretion of IFN $-\gamma$ has been reported to be an important mechanism for limiting viral replication (Muller et al., 1994; van den Broek et al., 1995a, 1995b). However, certain LCMV strains have been shown to be resistant to interferon mediated inhibition of replication (Moskophidis et al., 1994), and interferon may also act in a different way in LCMV compared to HBV infection, e.g. by countering CTL exhaustion (van den Broek et al., 1995a, 1995b; Wodarz et al., 1998) or enhancing MHC class I expression and antigen presentation in infected cells (Kagi et al., 1995b). Interferon in- LCMV infection may also not be produced by the LCMV-specific CTLs, but by unspecific immune mechanisms acting mainly early in the infectious process (Kagi et al., 1995b). Given that besides a lytic CTL response, the main alternative immune mechanisms are those acting on free virus particles, such as a neutralising antibody response or CTL-mediated inhibition of virus entry into arget cells, our models predict that perforinknockout mice will lose control of non-cytopathic viruses compared to perforin-competent mice. Further experiments could perhaps identify the dominant anti-LCMV immune response in the perforinknockout studies discussed above.

\section{CONCLUSIONS}

In conclusion, the hypothesis that CTL mediated lysis of infected cells is essential for controlling non-cytopathic viruses, whereas soluble immune factors are sufficient to control cytopathic viruses, may not be generally valid. While the models have shown that a lytic CTL response indeed controls the two types of viruses with very similar efficiencies, we have also demonstrated that with soluble immune mediators, it depends on the mechanism of action whether non-cytopathic viruses will be able to achieve significantly higher virus loads than cytopathic ones.

These results also have important implications for human immunodeficiency virus (HIV) infection. At the beginning of the infectious process at the time of seroconverison, relatively slowly replicating and non-cytopathic strains (macrophage tropic/NSI) predominate in the host (Tersmette et al., 1989; Schuitemaker et al., 1992; Connor and Ho 1994, Schuitemaker, 1994; Rudensey et al., 1995; Fouchier et al., 1996). As disease develops, faster replicating, more cytopathic strains ( $\mathrm{T}$ cell tropic/SI) emerge and this is associated with progression to full blown AIDS (Tersmette et al., 1989; Schuitemaker et al., 1992; Connor and Ho, 1994; Schuitemaker, 1994; Rudensey et al., 1995; Fouchier et al., 1996). Here we have shown that cytopathogenicity can be a major determinant of virus load and that fitness differences between cytopathic- and non-cytopathic viruses may depend on the immune responses acting on the virus population. These results may help to understand the selective mechanisms underlying the dominance of macrophage tropic less cytopathic strains at the beginning of the infection and the emergence of increasingly $\mathrm{T}$ cell tropic more cytopathic strains towards the end. This is explored further in a separate paper (Wodarz and Nowak, 1998).

\section{Acknowledgements}

We thank Paul Klenerman for helpful discussions.

\section{References}

Anderson, R. M. and May, R. M. (1979). Population biology of infectious diseases: Part 1. Nature, 280, 361-367.

Anderson, R. M. and May, R. M. (1991). Infectious diseases of humans. Dynamics and control. Oxford University Press.

DeBoer, R. J. and Perelson, A. S. (1998). Target cell limited and immune control models of HIV infection: a comparison. Journal of Theoretical Biology, 190, 201-214.

Bonhoeffer, S. and Nowak, M. A. (1997). Pre-existence and emergence of drug resistance in HIV-1 infection. Proceedings of the Royal Society of London, Seires B, 264, 631-637.

Bonhoeffer, S., May, R. M., Shaw, G. M. and Nowak, M. A. (1997a). Virus dynamics and drug therapy. Proceedings of the 
National Academy if Sciences of the United States of America, 94, 6971-6976.

Bonhoeffer, S., Coffin, J. M. and Nowak, M. A. (1997b). Human immunodeficiency virus drug

of Virology, 71, 3275-327.

of Wr. W (1994). HIV-1 entry and reverse transcription in

O'Brien, W. A. (1994). HIV-1 entry Biology, 56, 273-277.

macrophages. Journal of Leukocyte Bing, S., Zinkernagel, R. M.

van den Broek, M. F., Muller, U., Huang, S., Zin mice lacking type and Aguet, M. (1995a). Immune defence in mice lacal Reviews, $I$ and/or type

148, 5-18.
. Zinkernagel, R. M. (1995b). Antiviral defense in mice lackal of both alpha/beta and gamm

Virology, 69, 4792-4796.

Cavanaugh, V. J., Guidotti, L. G. and Chisarication in transInterleukin-12 inhibits hepatitis B virus replical

genic mice. Journal of Virology, 71, $3236-3243$. Chisari, F. V. (1997). Cytotoxic T cells and 1477

nal of Clinical Investigation, 99, 1472-1477.

Chisari, F. V. and Ferrari, C. (1995). Hepanology, 13, 29-60. pathogenesis. Annual Review of Immunology, 13, 29- K., Gallo,

Cocchi, F., Devico, A. L., Garzinodemo, A., Arya, S. K., Gal, MipR. C. and Lusso, P. (1995). Identification of RANressive factors 1-Alpha, and MIP-1-beta as the major HIV suppressive produced by CD8 ${ }^{+}$T-cells. Science, Human immunodeficiency

Connor, R. I. and Ho, D. D. (1994). Hured replicative capacity virus type 1 variants with increased before disease prodevelop during the asymptomatic stage befor. gression. Journal of Virology, 68, 4400-494). Introduction to Dimmock, N. J. and Primrose, S. B. (1994). Introductory Series, modern vi Blackwell.

S. (1994). Modelling HIV-infection

Essunger, P. and Perelson, A. S. (1994). Mournal of Theoretical Biolof $\mathrm{CD} 4^{(+)} \mathrm{T}$ cell sub ogy, 170, 367-391.

. Aropism and higher and Schuitemaker, $\mathrm{H}$. (1) cytopathicity for $\mathrm{CD}_{4}^{(+)}$T-cells compared to a non-syncyticum-inducing cell decline in vivo. mechanism for accele Virology, 219, 87-95.

Gallo, R. C. and Lusso, P. (1997). Chemokines and 12-17. tion. Current Opinion of Infectious Diseases, 10 . To to cure -

Guidotti, L. G. and Chisari, F. V. (1996). To kin. Current Opioptions in host-defense against nion in Immunology, 8, 478-483. $\mathrm{V}$ Ishikawa, T., Runkel, L. Guidotti, L. G., Ando, K., Hobbs, M. V., (1994a). Cytotoxic TSchreiber, R. D. and Chisari, F. V. (19)ene expression by a lymphocytes inhibit hepatitis- $\mathrm{B}$ virus gene. expreedings of the noncytolytic mechanism in transgenic mice. Proceed of America, National Academ

91, 3764-3768.

S. and Chisari, F. V. (1994b). Interuidotti, L. G., Guilhot, S. and Chisan down-regulate hepatitisleukin-2 and alpha/beta interferon dow tumor necrosis factorB virus gene-expression in-vivo by turways. Journal of Virodependent and factor-

$\log y, 68,1265-1270$

Gobs, M. V., Matzke, B., Gresser, Guidotti, L. G., Borrow, P., Hobsari, F. V. (1996a). Viral crossOldstone, M. B. A. and Chation of the hepatitis virus during talk - intracellular inactivation the liver. Proceedings of the an unrelated viral-infection of the United States of America, National Academ

93, 4589-4594. Ishikawa, T., Hobbs, M. V., Matzke, B.,
Guidotti, L. G.,
Schreiber, R. and Chisari, F. V. (1996b). Intracellular inactivation of the hepatitis-B virus by cytotoxic $\mathrm{T}$ lymphocytes. Immunity, 4, 25-36.

. Different roles for cytotoxic agi, D. and Hengarter of infections with cytopathic versus T-cells in the control of inrect Opinion in Immunology, 8, noncytopat

472-477. Kagi, D., Ledermann, B., Burki, K., Seiler, P. $\ddot{M}$. and HengartOlsen, K. J., Podack, E. R., Zinkernagel, R. M. and naturalner, H. (1994). Cytotoxicity mediated by killer-cells is greatly

Nature, 369, 31-37. $\quad$ Burki, K., Zinkernagel, R. M. and

Kagi, D., Ledermann, B., Burki, K., Zinkernaged cytotoxicity Hengartner, H. (1995a). Lymphocyte-mediated cynce. Immunoin-vitro and in-vivo - mechan. logical Reviews, 146, 95-115. Kagi, D., Seiler, P., Pavlovic, J., Lederm (1995b). The roles of kernagel, R. M. and Hengartner, H. ( cytotoxicity in protecperforin-dependent and Fas-dependent cytotoxicity in European tion against cytopathic and noncytopath.

Journal of Immunology, 25, 3256-3262. Kagi, D., Ledermann, B., Burki, K., Zinkernagel, R. M. and Hengartner, H. (1996). Molecular mechanisms of lymological protecmediated cytotoxicity and their role in immunological prology, tion and path

14, 207-232.

McLean, A. R. (1992). Mathematical-modeling of the immunization of populations. Reviews in Medical Virology, 2, 141-152. McLean, A. R. and Kirkwood, T. B. L. (1990) T-helper cell human-immunodeficiency-virus infection 147, 177-203.

clones. Journal of Theoretical Biology, $147,177-203$. Lehmann-Grube, F. (1971). Lymphocyti

Virology Monographs, 10, 1-173. Levy, J. A., Mackewitz, C. E. and Barker, E. (1996). Conto antiling HIV pathogenesis - the role of the nonology Today, 17, HIV respons

217-224. Michalak, T. 1., Pasquinelli, C., Guilhot, S. and Chery from acute (1994). Hepatitis-B virus persistence afterion, 93, 230-239. viral hepatitis. Journal of Clinical Investigation, 93,230 , R. M. Moskophidis, D., Lechner, F., Pircher, H. and Zimmunocompe(1993). Virus persistence in acutely infected imfector T cells. tent mice by exhaustion Nature, 362, 758-761. Nature, 362, D., Battegay, M., Bruendler, M., Laine, E., Gres-
Moskophidis, D., ser, I. and Zinkernagel, R. M. (1994). Resistance of and phocytic choriomeningitis virus to alpha/beta interfers. gamma interferon. Journal of Virology, Broek, M., Laine, E., Moskophidis, D., Battegay, M., van den Broek, M., Las). Role of Hoffmann-Rohrer, U. and Zinkernagel, R. M. (19 immunopathovirus and host variables in virus persistence or imournal of Genlogical disease caused by non eral Virology, 76, 381-391. . F. L., Hemmi, S., Pavlovic, J., Muller, U., Steinhoff, U., Reis, L. F. L., He94). Functional role of Zinkernagel, R. M. and Aguet, M. (1994). Funse. Science, 264, type-I and type-I

$1918-1921$

Nowak, M. A. and Bangham, C. R. M. (1996). Population dynamicsence, 272 74-79.

Rehermann, B., Ferrari, C. and Chisari, F. V. (1995). The cytotoxic T-lymphocyte response persists 22, 466-466. acute viral hepatitis. Hepatology, 22, 466-466. Chisari, F. V. Rehermann, B., Ferrari, C., Pasquinelli, C. and Chafter patients (1996). The hepatitis-B virus persists for decades after paient 
recovery from acute viral hepatitis despite active maintenance $1104-1108$.

Nature Medicine, 2

badensey, L. M., Kimata, J. T., Benveniste, R. E. baugh, J. (1995). Progression to AIDS ite, R. E. and Overated with changes in the replication in Macaques is associproperties of the simian immunodeficiency lation. Virology, 207, 528-542 Schuitemaker, H, Ko , 528-542.

deGoede, R. E. Y. Schattenkerk, J. K. M. E., Miedem, R. P., Lange, J. M. A., (1992). Biological phenotype of hum . and Tersmette, M virus type-1 clones at different of human immunodeficiency sion of disease is associated with a sift infection - progresto $T$-cell tropic virus populations. 1354-1360.

Schuitemaker,

tiators of infection and Macrophage-tropic HIV variants - inicyte Biology, 56, 218-224. pathogenesis. Journal of LeukoTersmette,

Lange, J. M. A., Schellekens Wolf, F., de Goede, R. D. Y., man, H. G. and Miedem, F. (1989). A., Goudsmit, J., Huisvirulent human immunodeficiency). Evidence of a role of the pathogenesis of anodeficiency virus (HIV) variants in studies on sequential HIV isolates. 2118-2125.
127

(1995). Posttranscription., Ishikawa, T. and Chisari, F. V. by cytotoxic T-lymphocyte-activate of hepatitis-B virus-RNA of the National Academy of Sciences of the Untes. Proceedings America, 92, 12398-12402 Sciences of the United States of

Walsh, C. M., Matloubian, M., Liu, C. C., Ueda, R., Kurahara,
C. G., Christensen, J. L., Huang, M. Ahmed, R. and Clark. Wuang, M. T. F., Young, J. D. E, mice lacking the perforin . R. (1994). Immune function in Academy of Sciences of the United Stangs of the National 10854-10858.

Wodarz 10858.

immune responses on the A. (1998). The effect of different HIV. Proceedings of the Royal Socion of virulent CXCR4 tropic press. Wodarz

Wodarz, D., Klenerman, P. and Nowak, M. A. (1998). Dynamics
of cytotoxic T-lymphocyte Royal Society of Londonte exhaustion. Proceedings of the Zanussi, S., Dandrea, M. Simes B, 265, 191-203.

aoli, P. (1996). Serum levels of R., Tirelli, U. and Depin HIV positive long-term survivors antES and MIP-1 alpha $A I D S, 10,1431-1432$.

Zinkernagel, R. M. (1996).

ence, 271, 173-178. 


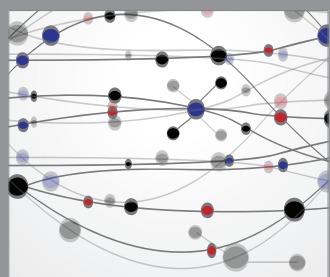

The Scientific World Journal
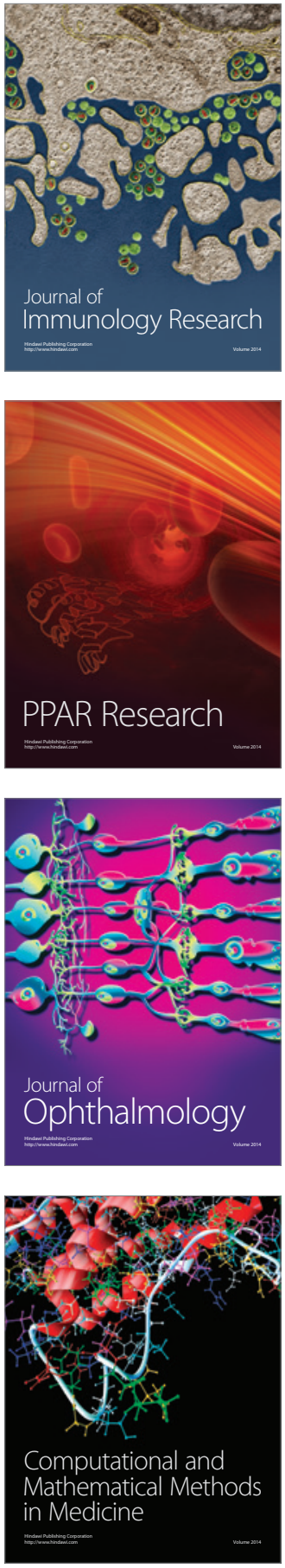

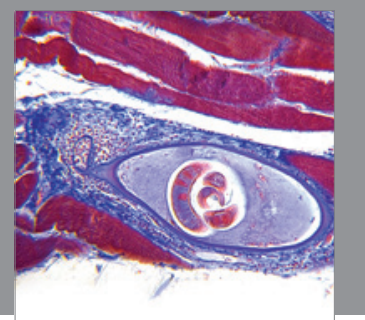

Gastroenterology

Research and Practice
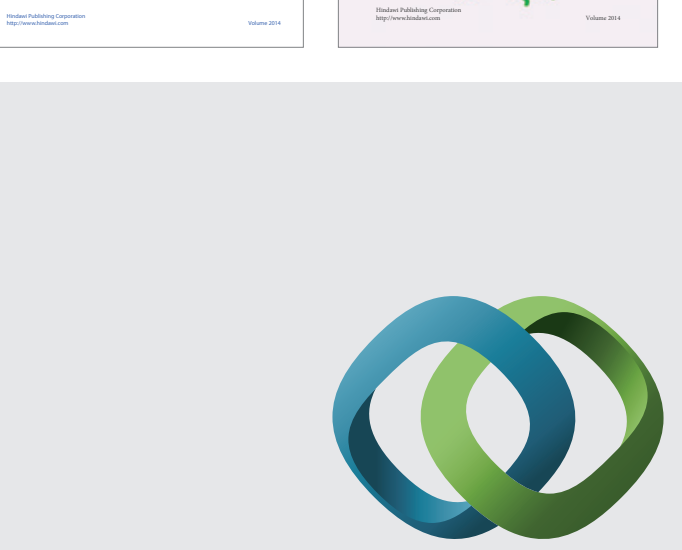

\section{Hindawi}

Submit your manuscripts at

http://www.hindawi.com
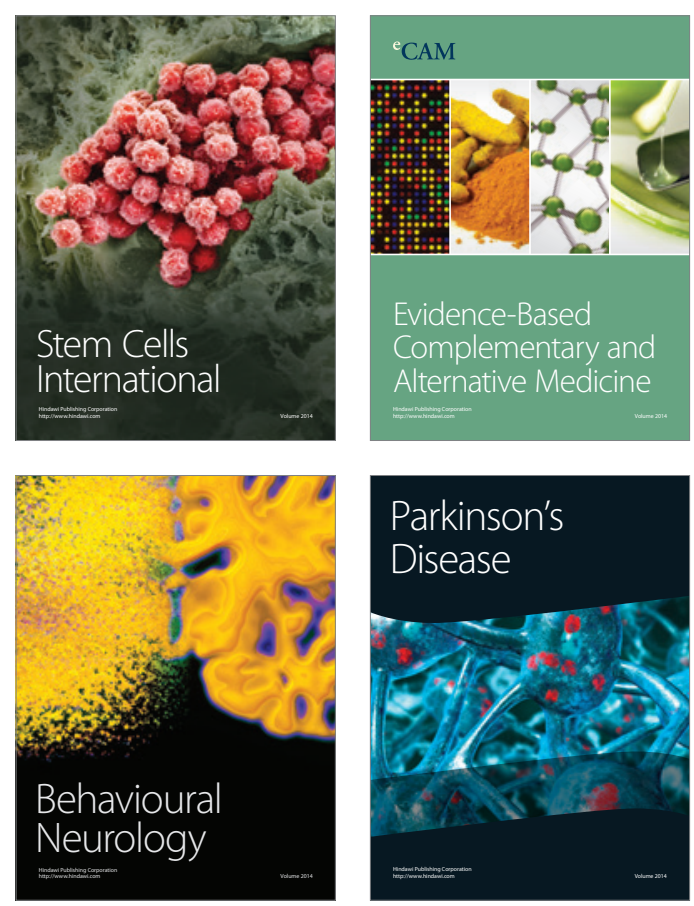

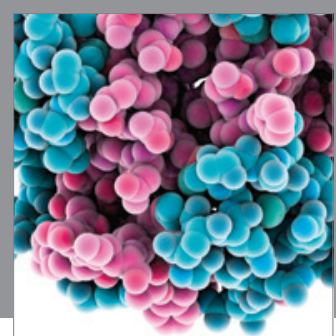

Journal of
Diabetes Research

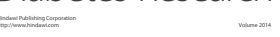

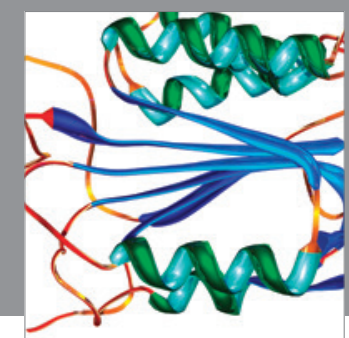

Disease Markers
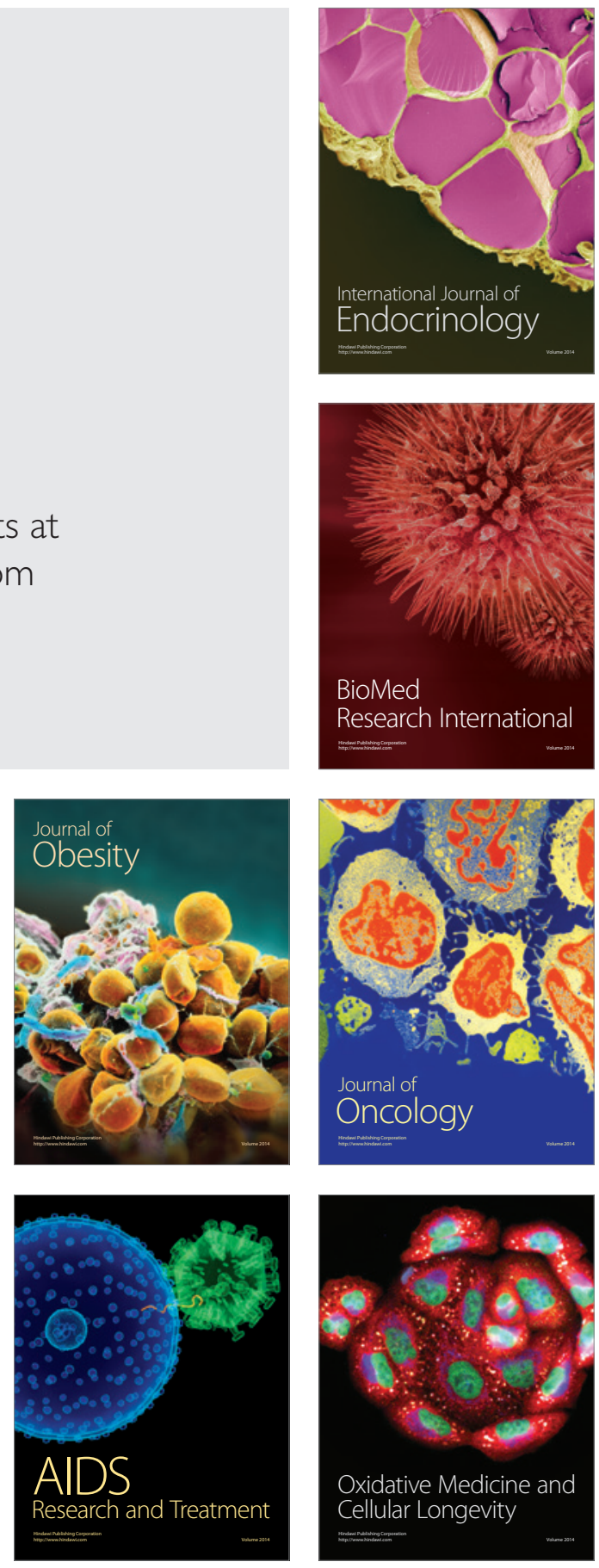